\title{
Introduction to Future Directions of Music Cognition proceedings
}

\author{
Joshua Albrecht ${ }^{1 \dagger}$ \\ Lindsay Warrenburg ${ }^{2}$ \\ Lindsey Reymore ${ }^{2}$ \\ Daniel Shanahan ${ }^{2}$ \\ ${ }^{1}$ Hugh A. Glauser School of Music, Kent State University, Kent, OH, USA \\ ${ }^{2}$ School of Music, Ohio State University, Columbus, OH, USA \\ Published 16 December 2021; https://doi.org/10.18061/FDMC.2021.0001 \\ † Corresponding author: jalbrec6@kent.edu
}

\begin{abstract}
The Future Directions of Music Cognition conference developed parallel to many of the societal changes brought about by the COVID-19 pandemic. The result was an innovative approach to online, remote conferencing. This proceedings journal reflects the research presented at the conference and speaker series, but the virtual nature of the conference also provides opportunities for this journal to be multimedia in new and exciting ways. Most of the articles in this journal are accompanied by an online multimedia presence, with articles linked to videos of the authors presenting, slides, and access to original data and stimuli. This article reviews the story of how the conference and speaker series developed during the pandemic, some of the achievements of the conference, and how the journal is structured.
\end{abstract}

\section{Retrospective: How we got here}

It takes a certain degree of boldness to invoke the idea of Future Directions when starting a new project. Research develops quickly, especially in the sciences. Rapidly developing technology consistently drives methodological innovations, and new knowledge is constantly being generated through the collection of new experimental data. The field of music cognition is a case in point, with increasing expansion in recent years, reflected both in new research questions and ever deepening subfield specializations.

Nevertheless, in the summer of 2019, Lindsay Warrenburg, Lindsey Reymore, Daniel Shanahan, and I sat down to begin planning the Future Directions of Music Cognition conference, originally scheduled for the summer of 2020 at The Ohio State University in Columbus, Ohio. The development of the conference was motivated precisely by these recent rapid developments in the field. Given the increasing size and scope of the field, one goal for the conference was to provide a resource for researchers wanting to evaluate methods and technologies outside of their primary area of specialization. We also wanted to create new vehicles for participants to engage in dialogue about the field and its future directions while having a centralized forum to review current methodological innovations and trends.

To accomplish these goals, the conference was designed in part as a deep dive into five popular subspecialities to review the current state of the field and possibilities for future directions of research. These five topics - corpus studies, emotion, rhythm and meter, timbre, and pedagogy - would each have been featured for one of the conference's five days. Each day would have featured a blend of activities related to the sub-discipline, including a keynote presentation and a methodology workshop led by an expert on the topic, presentations and posters on the topic by conference attendees, and concentrated working groups led by the expert to design and potentially run studies on that topic to be presented at the end of the conference.

Of course, it's always challenging to accurately predict the future. But 2019 might have been one of the single worst times in recent history to make long term plans. After we had secured our five topic experts, received 114 submissions for presentations and posters, and recruited a panel of 25 expert reviewers to recommend submissions for acceptance, the global COVID-19 pandemic broke out, followed by an unprecedented shutdown of the worldwide economy and the near elimination of face-to-face academic discourse. Consequently, our original plans for an inperson conference were first postponed and then redirected entirely.

\section{The new shape of the conference}

In grappling with the realities of a worldwide pandemic, we quickly decided that it would be better to 
reimagine the conference rather than to simply substitute subpar virtual imitations for our original inperson plans. Given the realities of screen fatigue, we were also wary of a week-long online conference schedule. The challenges of moving online, however, also brought opportunities for us to more fully incorporate some of our values into the structure of the conference. For example, our organizing principles included free and open access to conference resources, an emphasis on advancing opportunities for young scholars, and increasing the inclusivity of our community.

With these values and constraints in mind, Future Directions of Music Cognition was reimagined as two complementary events. The first of these events was an online conference, held March 6-7, 2021. This conference featured the originally accepted talk and poster presentations in sessions in topical groupings, roughly corresponding to the original conference. The two-day long conference featured 49 long talks of 12 minutes and 21 lightning talks ("posters") of 5 minutes. A full schedule can be found at the MASCATS website (MASCATS, March 6-7 Schedule, 2021).

To increase accessibility, conference registration was free and open to the public. With travel costs eliminated, over 100 attendees from around the world were able to participate in the two-day conference. In our efforts to support young scholars, we were able to honor six exemplary student presentations with awards totaling \$1,800 (MASCATS, Student Awards, 2021). The conference also featured several opportunities for participants to socialize. These included video conferencing sites available during coffee and lunch breaks, and three sessions in which participants were randomly paired up for five-minute social sessions. A Slack channel, dedicated to conference chat about sessions and presentations, happened in real time and retrospectively (Slack, 2021). Over 500 people from 16 different time zones participated on this Slack channel, consistent with our goal of expanding access to the conference. Finally, through hosting with the Open Science Framework, we have uploaded all conference presentations for permanent and free online access, including both pre-recorded presentations and live recordings at the conference (Open Science Framework, Future Directions of Music Cognition March Conference, 2021). Readers of this proceedings journal are encouraged to supplement their reading with videos of the authors presenting, in which sound examples and other multimedia enrichment are often included.
The second component of Future Directions was a speaker series. The shift to a virtual conference allowed us to greatly expand the number of experts involved. Fifteen presentations were delivered weekly in a synchronous online format to our worldwide audience between February and May 2021. Consistent with our goal of opening up resources to a wide audience, over 1,200 people signed up for some aspect of the conference, representing 427 universities and 32 industries in 46 countries on 6 continents. Topics aligned with the original five sub-specialties within music cognition along with several other topic areas, such as neuroscience, music theory, music industry and technology, genetics, and sociology. The speaker series included thirteen hour-long keynote talks and two additional initiatives. The first initiative, an "alt-ac" panel discussion, featured four researchers pursuing careers outside of academia and provided a variety of resources for those interested in following alternative career paths beyond the traditional academic route (Open Science Framework, Alt-ac Resources, 2021). The second initiative was an interactive town hall discussion centered on the future role of anti-racism efforts in the Society for Music Perception and Cognition (Gather Town, 2021). Speaker series presentations were recorded and uploaded to the Open Science Framework, and will be stored in perpetuity as a freely accessible resource available to researchers and the public (Open Science Framework, Future Directions of Music Cognition - Speaker Series, 2021).

Together, the online virtual conference and the speaker series represent a significant effort of both established and new scholars to grapple with the current and future state of our scientific communities and research in light of the evolving nature of our field and new methods and theories.

\section{How to read this journal}

The structure of the proceedings journal reflects the conception of the conference. There are seven main sections, starting with articles by David Huron (pp. 524) and Psyche Loui (pp. 25-31), two of the speaker series presenters. Huron's paper, "On the future of music research," is a wide ranging article on the nature of music research, examining some first principles, reviewing some of the most significant achievements, and looking forward to new fruitful directions music research may take, followed by a reproduction of the question and answer period after his talk. Loui's paper, 
"Use-inspired music cognition: Designing cognitively informed musical interventions for the brain," casts light on the future of music cognition research by reviewing and drawing lessons from the early days of the field, arguing for a deeper integration of pure music cognition research with practical applications of musicbased interventions that can genuinely help people and make the world a better place.

Following this first section, the remaining sections reflect the conference's contributions in six of the music cognition sub-disciples represented by the conference, including corpus studies, music and emotion, pedagogy, music perception, rhythm and meter, and timbre. Each section consists of full-length articles organized by first author last name. Sections end with the published abstracts for that sub-discipline. Finally, the journal ends with an editorial epilogue that reflects on how the Future Directions of Music Cognition conference, speaker series, and proceedings interact with some of the societal changes that have happened between the original conception of the conference and its conclusion as a way of looking forward to what future directions the field may take.

As mentioned above, all conference presentations were recorded and posted in perpetuity on the Open Science Framework's (OSF) Future Directions of Music Cognition - March Conference web page (2021). Speaker series talks are also freely available at OSF's Future Directions of Music Cognition Speaker Series website (2021). Most of the articles in this journal were also presented at the conference, and so readers can watch video presentations on OSF. Additionally, several OSF pages also include slides of presentations, original data from studies, stimuli used, or other background materials. DOI links for each presentation are included in the header of each article below author information. Interested readers are encouraged to further examine online content.

Finally, as of this publication date the conference Slack channel (2021) remains available, with separate channels for each session. Visitors to the Slack channel can review the history of questions, answers, and comments associated with each paper. By joining the Slack channel, readers may even post their own questions to authors and continue public dialogue about the research in this journal.

\section{References}

Gather Town. (2021). Town Hall on Anti-Racism in Music Cognition.

https://gather.town/app/BYatfy3d9rD3cwA0/FD antirac ismTH

MASCATS. (2021). March 6-7 schedule. http://org.osu.edu/mascats/march-6-7-schedule/

MASCATS. (2021). Student Awards. http://org.osu.edu/mascats/student-awards

Open Science Framework. (2021). Alt-ac Resources Future Directions. https://osf.io/gbcp6

Open Science Framework. (2021). Future Directions of Music Cognition - March Conference. https://osf.io/meetings/FD2021Conf/

Open Science Framework. (2021). Future Directions of Music Cognition - Speaker Series. https://osf.io/meetings/FD2021Sp

Slack. (2021). Future Directions of Music Cognition. futuredirecti-nvq6273.slack.com 